\title{
SARS-CoV-2 viral-load distribution reveals that viral loads increase with age: a retrospective cross-sectional cohort study
}

\author{
Sjoerd Euser, ${ }^{1 *}$ Sem Aronson, ${ }^{1,2}$ Irene Manders, ${ }^{1,3}$ \\ Steven van Lelyveld, ${ }^{2}$ Bjorn Herpers, ${ }^{1}$ Jan Sinnige, ${ }^{1}$ Jayant Kalpoe, $^{1}$ \\ Claudia van Gemeren, ${ }^{4}$ Dominic Snijders, ${ }^{5}$ Ruud Jansen, ${ }^{1}$ \\ Sophie Schuurmans Stekhoven, ${ }^{2}$ Marlies van Houten, ${ }^{6}$ Ivar Lede, ${ }^{7}$ \\ James Cohen Stuart, ${ }^{8}$ Fred Slijkerman Megelink, ${ }^{9}$ Erik Kapteijns, ${ }^{10}$ \\ Jeroen den Boer, ${ }^{1}$ Elisabeth Sanders, ${ }^{11,12}$ Alex Wagemakers ${ }^{1,+}$ and \\ Dennis Souverein (1) ${ }^{1, \dagger}$
}

\begin{abstract}
'Department of Epidemiology, Regional Public Health Laboratory Kennemerland, Haarlem, The Netherlands, ${ }^{2}$ Department of Internal Medicine, Spaarne Gasthuis, Hoofddorp/Haarlem, The Netherlands, ${ }^{3}$ Department of Infectious Diseases, Public Health Service Kennemerland, Haarlem, The Netherlands, ${ }^{4}$ Intensive Care Unit, Spaarne Gasthuis, Hoofddorp/Haarlem, the Netherlands, ${ }^{5}$ Department of Pulmonary Disease, Spaarne Gasthuis, Hoofddorp/Haarlem, the Netherlands, ${ }^{6}$ Department of Pediatrics, Spaarne Gasthuis, Hoofddorp/Haarlem, the Netherlands, ${ }^{7}$ Department of Medical Microbiology, Comicro BV Medical Microbiology, Hoorn, The Netherlands, ${ }^{8}$ Department of Medical Microbiology, Noordwest Ziekenhuisgroep, Alkmaar, The Netherlands, ${ }^{9}$ Department of Infectious Diseases, Public Health Service Hollands Noorden, Alkmaar, The Netherlands, ${ }^{10}$ Department of Pulmonary Disease, Rode Kruis Ziekenhuis, Beverwijk, The Netherlands, "Department of Immunology and Infectious Diseases, Wilhelmina Children's Hospital, University Medical Center Utrecht, Utrecht, The Netherlands and ${ }^{12}$ Center for Infectious Disease Control, National Institute for Public Health and the Environment, Bilthoven, The Netherlands
\end{abstract}

*Corresponding author. Regional Public Health Laboratory Kennemerland, Boerhaavelaan 26, 2035 RC, Haarlem, The Netherlands. E-mail: s.euser@streeklabhaarlem.nl

${ }^{\dagger}$ Shared senior authors.

Received 23 April 2021; Editorial decision 23 June 2021; Accepted 28 June 2021

\begin{abstract}
Background: Describing the SARS-CoV-2 viral-load distribution in different patient groups and age categories.

Methods: All results from first nasopharyngeal (NP) and oropharyngeal (OP) swabs from unique patients tested via SARS-CoV-2 reverse transcriptase polymerase chain reaction (RT-PCR) collected between 1 January and 1 December 2020 predominantly in the Public Health Services regions Kennemerland and Hollands Noorden, province of North Holland, the Netherlands, were included in this study. SARS-CoV-2 PCR crossing-point (Cp)-values were used to estimate viral loads.
\end{abstract}


Results: In total, 278455 unique patients were tested, of whom 9.1\% ( $n=25.374)$ were SARS-CoV-2-positive. PCRs performed by Public Health Services $(n=211914)$, in which sampling and inclusion were uniform, revealed a clear relation between age and SARSCoV-2 viral load, with especially children aged $<12$ years showing lower viral loads than adults ( $\beta$ : $-0.03,95 \%$ confidence interval: -0.03 to $-0.02, p<0.001)$, independently of sex and/or symptom duration. Interestingly, the median Cp-values between the $>79$ - and $<12$-year-old populations differed by more than four PCR cycles, suggesting an $\sim 16$-fold difference in viral load. In addition, the proportion of children aged $<12$ years with a low load ( $\mathrm{Cp}$-value $>30)$ was higher compared with other patients $(31.1 \%$ vs $17.2 \%, p$-value $<0.001)$.

Conclusions: In patients tested by Public Health Services, SARS-CoV-2 viral load increases with age. Further studies should elucidate whether the lower viral load in children is indeed related to their suggested limited role in SARS-CoV-2 transmission. Moreover, as rapid antigen tests are less sensitive than PCR, these results suggest that SARS-CoV-2 antigen tests have lower sensitivity in children than in adults.

Key words: SARS-CoV-2, viral-load distribution, age, COVID-19

\section{Key Messages}

- SARS-CoV-2 viral load increases with age.

- Median crossing-point (Cp)-values (viral load) between the oldest ( $>79$ years) and youngest ( $<12$ years) population differed by over four polymerase chain reaction (PCR) cycles, suggesting an $\sim 16$-fold difference in viral load.

- Rapid antigen tests are less sensitive than PCR, suggesting that SARS-CoV-2 antigen tests have lower sensitivity in children than in adults.

\section{Introduction}

Since the start of the COVID-19 pandemic, molecular testing of respiratory samples by reverse transcriptase polymerase chain reaction (RT-PCR) has been the primary method to diagnose

SARS-CoV-2. ${ }^{1}$ Although SARS-CoV-2 RT-PCR results are in general reported in a qualitative manner (positive or negative), the quantitative test result [cycle threshold (Ct) or crossing point $(\mathrm{Cp})$, which indicates the viral load in a sample] offers additional insights into e.g. SARS-CoV-2 transmission dynamics. Individual viral-load kinetics show a sharp increase in viral load in the earliest (mostly presymptomatic) stages of the infection, followed by a gradual decline. ${ }^{2}$ Interestingly, whereas viral cultures are mostly positive in samples with high viral load (Ct-value $<25$ ), samples with a low viral load hardly show any potential for viral cultivation $(<3 \%$ at $\mathrm{Ct}$ value 35$)$, suggesting lower risk of transmission. ${ }^{3}$

One of the problems of studying SARS-CoV-2 viral loads in respiratory samples is the lack of comparability of $\mathrm{Ct}$ - or $\mathrm{Cp}$-values derived from different laboratories, as these are assay- and method-specific. ${ }^{4}$ This issue complicates the evaluation of SARS-CoV-2 viral loads in respiratory samples derived from large patient populations where often multiple laboratories are involved in analysing these samples.

In this report, we describe the SARS-CoV-2 viral-load distribution of all routinely collected SARS-CoV-2-positive respiratory samples from a single large regional laboratory in the Netherlands, enabling us to evaluate the distinction between different patient groups [hospitalized patients, general-practitioner (GP) patients, nursing-home patients, healthcare workers, patients tested in Public Health testing facilities] and age categories with respect to viral-load distribution and the duration of symptoms.

\section{Methods}

\section{Setting, study design and participants}

The Regional Public Health Laboratory Kennemerland, Haarlem, the Netherlands, performs SARS-CoV-2 RT-PCR testing for $>800000$ inhabitants, including healthcare workers (HCWs), patients of four large teaching hospitals, patients of $>600$ GPs, 90 nursing-home organizations and those who are tested because of mild symptoms in Public Health testing facilities set up by the Public Health Services 
Kennemerland (PHS Kennemerland) and Hollands Noorden (PHS Hollands Noorden). Here, we report the SARS-CoV-2 RT-PCR results from nasopharyngeal (NP), oropharyngeal (OP) and combined swabs (first samples from unique patients only) that were analysed between 1 January and 1 December 2020, using the RT-PCR based on the presence of the E-gene. ${ }^{1}$ Cp-values were calculated using Lightcycler 480 1.5.1 software (Roche diagnostics, Basel, Switzerland). Swabs were derived from GP patients, hospital patients and hospital HCWs, nursing-home residents and nursing-home HCWs, and the majority were from Public Health testing facilities. As Public Health testing facilities employed a uniform sampling (combined oro- and nasopharynx) and inclusion policy, these PCRs were included for further analyses. Symptoms were required for Public Health testing in all test facilities; however, between 13 August and 13 September, returning travellers without a need for symptoms were also tested at Amsterdam Airport Schiphol. For several months during the testing period, the national testing policy required children to also have severe symptoms (dyspnoea or fever) or a positive contact to be included for testing (Supplementary Text, available as Supplementary data at IJE online).

The Public Health Service Kennemerland, which is responsible for the data collection and reporting of new COVID-19 cases on a national level and performs contact tracing for these cases in the Kennemerland region, provided the date of the first onset of disease for a subset of patients living in the Kennemerland region.

\section{Statistical analysis}

Descriptive statistics were used to present the data: continuous variables were presented as median [interquartile range (IQR)], categorical variables were presented as number $(\%)$. Comparisons of continuous variables were made using Mann-Whitney U tests; for categorical variables, Chi-square tests were used. The Kruskal-Wallis test, combined with post-hoc pairwise comparisons with Bonferroni correction for multiple testing, was used to investigate differences in viral load between different patient or age groups. Linear-regression analyses were used to analyse the relationship between age and viral load allowing additional adjustment for potentially confounding variables. Statistical analyses were performed using $\mathrm{R}$ and RStudio ( $R$ version 4.0.3), packages tidyverse, sf and broom.

\section{Results}

Between 1 January and 1 December, 278455 unique patients were tested, of whom the results of the first PCR were included. When patients were tested more than once, only the first positive PCR (when available) or the first negative PCR were included. Overall, 9.1\% $(n=25$ 374) samples were SARS-CoV-2-positive. Figure 1 shows the geographical distribution of the total number of positive unique patients for the period 1 January to 1 December 2020 in the Kennemerland region and adjacent areas.

\section{Viral-load distribution in different patient populations}

Comparison of the number of patients tested with the SARS-CoV-2 RT-PCR between the first (1 January to 31 July) and second (1 August to 1 December) waves of COVID-19 patients reveals a shift in the number of tested patients from different patient populations (Table 1). In the first wave, a relatively large proportion of tests $(26.6 \%)$ was performed for hospital patients, as compared with $0.8 \%$ in the second wave. The vast majority of samples $(80.9 \%)$ in the second wave were derived from Public Health testing facilities.

In addition, the distribution of Cp-values (with higher Cp-values indicating a lower viral load) of respiratory samples showed lower viral loads for samples collected during the first wave compared with samples collected during the second wave [median Cp-value (IQR): 28.4 (8.1) vs 25.7 (6.0), Mann-Whitney U test $p$-value $<0.001$ ] (Table 1). There were also remarkable differences in Cp-value distributions between patient populations who were tested in the first wave (Figure 2A) and the second wave of COVID-19 patients (Figure 2B). Analyses (Kruskal-

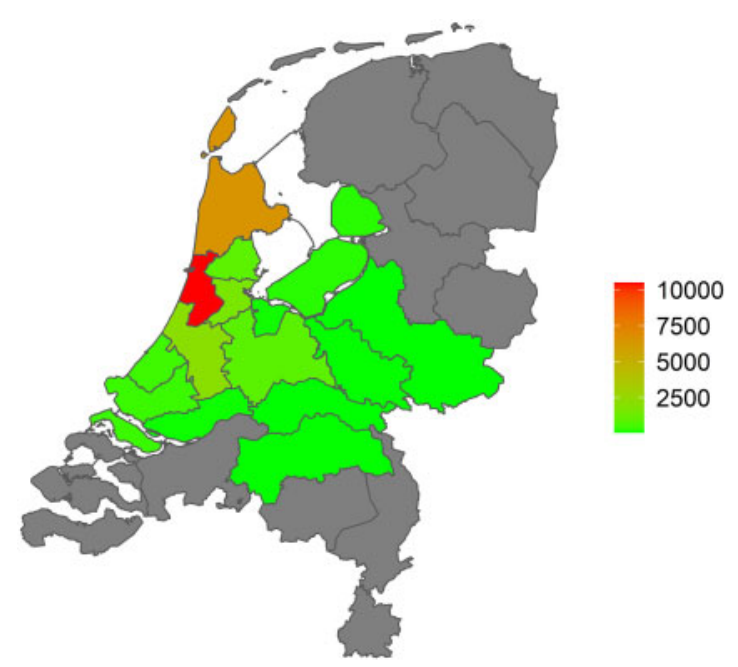

Figure 1 Geographical distribution of the total number of SARS-CoV-2 PCR-positive unique patients $(n=25374)$ tested in the Regional Public Health Laboratory Kennemerland for the period of January to December 2020.

Data are presented for Public Health Services areas; darker colours represent higher numbers of patients. The majority of patients lived relatively close to the laboratory, located in the red-coloured area. 
Wallis) indicated differences in viral load between these patient populations in both the first $(p$-value $<0.001)$ and second $(p$-value $=0.001)$ waves. Pairwise post-hoc comparisons (with a Bonferroni-corrected $p$-value) showed higher viral loads in the following patient populations in the first wave: Public Health (higher viral load) vs GP patients (lower viral load) ( $p$-value $=0.022)$, Public Health vs admitted hospital patients ( $p$-value $=0.001)$, Public Health vs not admitted hospital patients $(p$-value $<0.001$ ), Public Health vs nursing-home HCWs ( $p$-value $<0.001$ ), nursing-home residents vs not admitted hospital patients ( $p$-value $<0.001)$, nursing-home residents vs admitted hospital patients ( $p$-value $=0.007$ ) and nursing-home residents vs nursing-home HCWs ( $p$-value $=0.016$ ). In the second wave, nursing-home residents vs other groups ( $p$-val$\mathrm{ue}=0.004)$ and GP patients vs other groups ( $p$-val$\mathrm{ue}=0.008$ ) showed higher viral loads (Supplementary Tables S1 and S2, available as Supplementary data at IJE online).

\section{Viral-load distribution across age and gender categories}

The relationship between patient age and SARS-CoV-2 viral load was investigated in the Public Health testing facility samples collected between 1 January and 1 December $(n=211914)$, which was considered to be a relatively consistent population with respect to the performed sampling procedure (a combined NP/OP swab by similarly trained professionals) and patient characteristics. In total, 8.6\% $(n=18$ 290) samples were SARS-CoV-2-positive. Patients were categorized into different age categories: age $<12$ years (primary-grade school); 12-17 years (high school); 18-29 years; $30-49$ years; $50-59$ years; $60-69$ years; $70-$ 79 years; age $>79$ years. The number of available tests in each age category and the proportion of positive tests are shown in Table 2. Distribution of the SARS-CoV-2 Cp-values for these age categories is shown in Table 2 and in Figure 3. These data showed that both the proportion of positive tests as well as the SARS-CoV-2 viral load increase with age. The proportion positive tests was $4.3 \%$ in children aged $<12$ years compared with $8.7 \%$ in all other patients (Chi-square $p$-value $<0.001$ ). Analyses using a Kruskal-Wallis test indicated differences in viral load between the age categories ( $p$-value $<0.001$ ). Pairwise posthoc comparisons (with a Bonferroni-corrected $p$-value) showed differences between almost all age categories, except for those within higher age groups (Supplementary Table S3, available as Supplementary data at IJE online). In addition, the proportion of patients with a Cp-value of $>30$ was highest in the younger age groups. For example, the proportion of children aged $<12$ years with a $\mathrm{Cp}$-value 


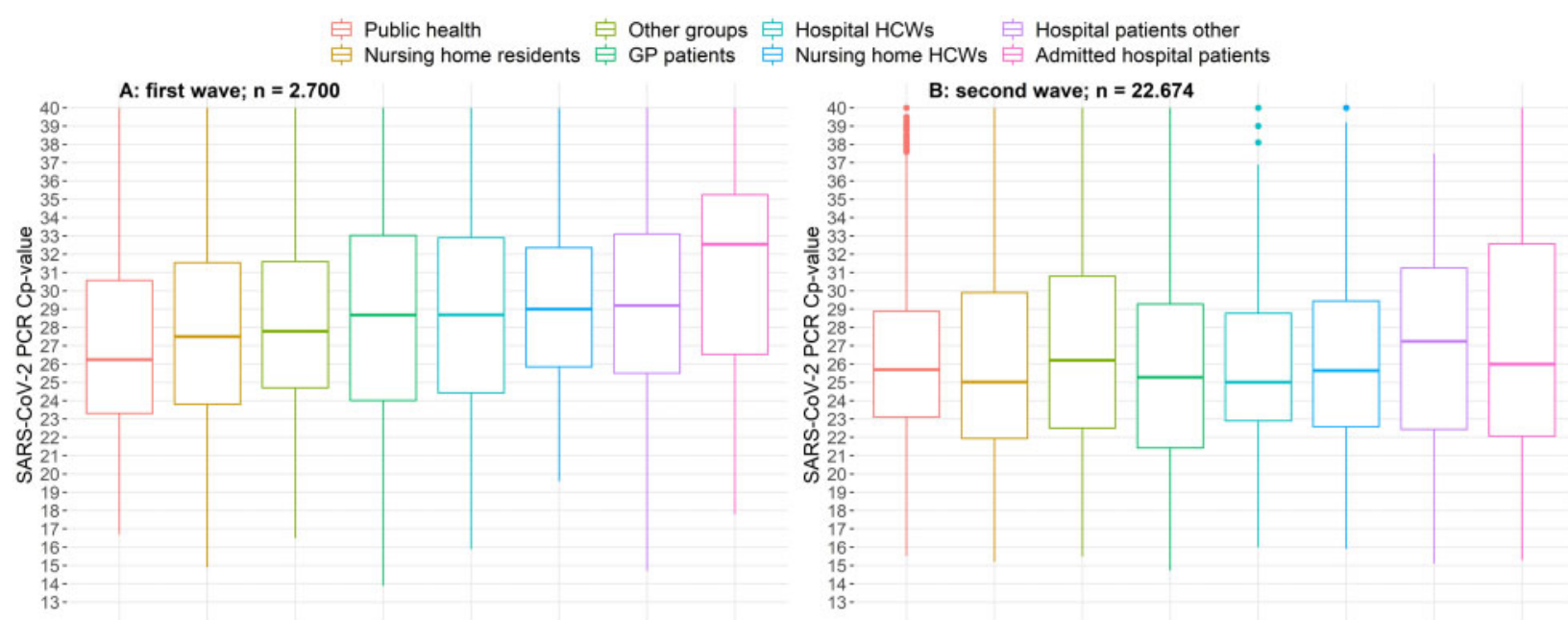

Figure 2 Distribution of SARS-CoV-2 polymerase chain reaction crossing-point (Cp)-values within different patient populations in the first (Panel $A$, $n=2700$ ) and second (Panel B, $n=22674$ ) waves of the COVID-19 epidemic in the Kennemerland region and adjacent areas

Each box corresponds to one specific patient population that was routinely tested in the period 1 January to 31 July (Panel A) or 1 August to 1 December (Panel B). Data are presented as box-and-whisker plots with the central box covering the interquartile range with the median $\mathrm{Cp}$-value indicated by the line within the box. The whiskers extend to the minimum and maximum values within 1.5 interquartile ranges of the quartiles; more extreme values are plotted individually. Higher $\mathrm{Cp}$-values indicate lower viral loads, as they describe the number of PCR cycles at which the amplification signal crosses the fluorescence threshold.

Table 2 Crossing-point (Cp)-value characteristics for different age groups in Public Health patients

\begin{tabular}{lcccccc}
\hline Age groups (years) & \multicolumn{5}{c}{ Cp-values } \\
\cline { 2 - 6 } & No. tested $(\%)$ & No. positive $(\%)$ & Cp-value (IQR) & Cp <20 no. $(\%)$ & Cp 20-30 no. $(\%)$ & Cp >30 no. $(\%)$ \\
\hline$<12$ & $5506(2.6)$ & $238(4.3)$ & $28.7(5.0)$ & $0(0)$ & $164(68.9)$ & $74(31.1)$ \\
$12-17$ & $22344(10.5)$ & $1589(7.1)$ & $26.9(5.7)$ & $33(2.1)$ & $1181(74.3)$ & $375(23.6)$ \\
$18-29$ & $47255(22.3)$ & $4372(9.3)$ & $26.0(5.6)$ & $132(3.0)$ & $3424(78.3)$ & $816(18.7)$ \\
$30-39$ & $41533(19.6)$ & $2771(6.7)$ & $25.9(5.7)$ & $61(2.2)$ & $2217(80.0)$ & $493(17.8)$ \\
$40-49$ & $34958(16.5)$ & $3068(8.8)$ & $25.5(5.6)$ & $92(3.0)$ & $2487(81.1)$ & $489(15.9)$ \\
$50-59$ & $30095(14.2)$ & $3466(11.5)$ & $25.0(5.3)$ & $165(4.8)$ & $2829(81.6)$ & $472(13.6)$ \\
$60-69$ & $19225(9.1)$ & $1751(9.1)$ & $25.3(5.8)$ & $78(4.5)$ & $1363(77.8)$ & $310(17.7)$ \\
$70-79$ & $8716(4.1)$ & $800(9.2)$ & $24.8(5.9)$ & $48(6.0)$ & $634(79.2)$ & $118(14.8)$ \\
$>79$ & $2282(1.1)$ & $235(10.3)$ & $24.6(5.4)$ & $13(5.5)$ & $195(83.0)$ & $27(11.5)$ \\
Total & $211914(100.0)$ & $18290(8.6)$ & $25.7(5.8)$ & $622(3.4)$ & $14494(79.2)$ & $3174(17.4)$
\end{tabular}

Data are presented as no. $(\%)$ or median. IQR, interquartile range.

of $>30$ was higher compared with the other patients $(31.1 \%$ vs $17.2 \%$, Chi-square $p$-value $<0.001)$. Interestingly, the median $\mathrm{Cp}$-values had a $>4 \mathrm{Cp}$-value difference between the oldest ( $>79$ years) and youngest ( $<12$ years) population, suggesting an $\sim 16$ times difference in viral load. Additional data on the viral load distribution in age groups over time (March to December) are presented in Supplementary Table S4 (available as Supplementary data at IJE online). These showed that the relationship between age and viral load was seen in all months, with a clear relationship in the months of August to December (when the majority of tests were performed). To evaluate the differences between patients from the different Public Health Services, additional analyses on the relationship between age groups and viral load were performed for each Public Health Service separately (Supplementary Table S5, available as Supplementary data at IJE online) as well as for the returning travellers between 13 August and 13 September, who were tested at Amsterdam Airport Schiphol $[n=6033$ of whom 74 $(1.2 \%)$ travellers tested positive]. These analyses showed that the relationship between age and viral-load distribution was independent of the Public Health Service location.

When the non-Public Health Service SARS-CoV-2-positive samples (derived from all other patient populations) were analysed separately $(n=6931)$, there was a 


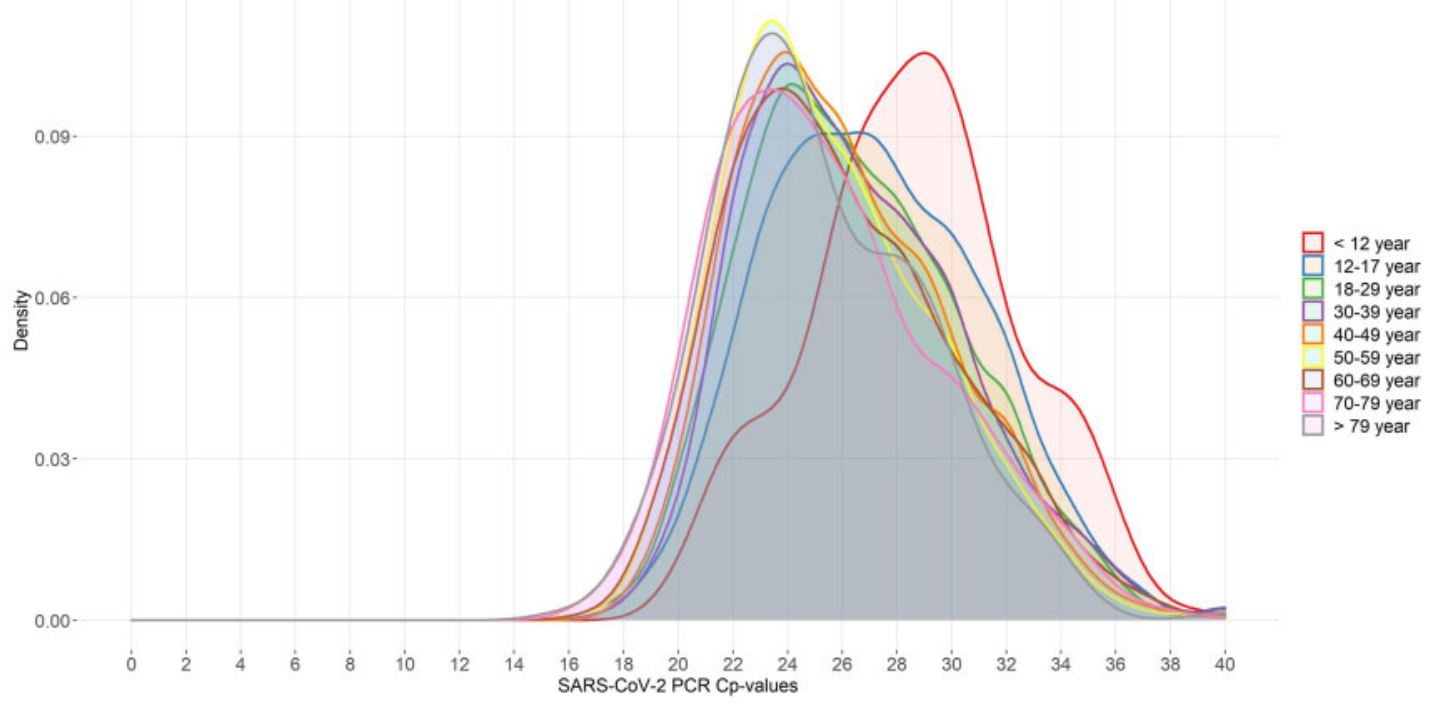

Figure 3 Distribution of SARS-CoV-2 polymerase chain reaction crossing-point (Cp)-values within different age groups $(n=18290)$ of patients tested in a Public Health setting.

Each colour corresponds to one specific age group that was routinely tested in the period 1 January to 1 December. For each group, the frequency of the reported $\mathrm{Cp}$-values was used to calculate a density score of which the area under the curve sums to 1 .

relationship between age and viral load present in the OP swabs [linear regression, $\beta:-0.01,95 \%$ confidence interval $(\mathrm{CI}):-0.02$ to $0.00, p$-value $<0.002)$ but not in the NP swabs $(\beta: 0.00,95 \% \mathrm{CI}:-0.02$ to $0.00, p$-value $=0.168)$ (Supplementary Table S6, available as Supplementary data at IJE online).

In a subset of 7300 samples from SARS-CoV-2 PCRpositive Public Health patients [mean (SD) age: 40.7 (17.8) years, $48.8 \%$ males] for whom the time between the onset of symptoms and testing was known [median (IQR) was: 2 (3) days], linear-regression analyses were performed. These analyses showed a relationship between increasing age and decreasing Cp-values: $\beta:-0.02,95 \%$ CI: -0.03 to $-0.02, p<0.001]$, independently of the time between the onset of symptoms and testing and sex (adjusted $\beta:-0.03,95 \%$ CI: -0.03 to $-0.02, p<0.001)$. In addition, when the relationship between patient sex and SARS-CoV-2 viral load was investigated (in the 7300 SARS-CoV-2 PCR-positive Public Health testing facility samples), men showed a slightly higher viral load as compared with women [median Cp-value (IQR): 25.5 (5.9) vs 25.8 (5.7), Mann-Whitney $\mathrm{U}$ test $p$-value $=0.015$ ] but, when this was adjusted for age and the time between the onset of symptoms and testing in the linear-regression analyses, no clear relationship between sex and viral load was seen (adjusted $\beta: 0.15,95 \%$ CI: -0.03 to 0.33 , $p=0.096$ ) anymore. Finally, a relationship was found between viral load and the time between the onset of symptoms and testing (adjusted $\beta: 0.45,95 \%$ CI: 0.41 to 0.49 , $p<0.001$ ), independent of age and sex.

\section{Discussion}

To our knowledge, this is the first study to evaluate SARSCoV-2 viral-load distributions in a large number of patients from different patient categories. Our data present a clear relationship between age and SARS-CoV-2 viral load, with children $(<12$ years) showing lower viral loads independent of sex and symptom duration. In addition, we observed that testing for COVID-19 using the SARS-CoV2 RT-PCR in a large Dutch region shifted between the first wave (1 January to 31 July) and second (1 August to 1 December) waves of identified COVID-19 patients with respect to the populations of patients who were tested and the SARS-CoV-2 viral-load distribution, with higher viral loads seen in samples analysed in the second wave. The shift in tested patients from different patient populations between the first and second waves was influenced by the national testing policy that was primarily focused on hospital patients in the first wave (due to limited testing capacity) and gradually moved to testing all patients with COVID-19-related symptoms (in large Public Health testing facilities) during the second wave. This could also have resulted in a more rapid diagnosis (since the onset of symptoms) for patients tested in the second wave resulting in the higher viral load detected in the second-wave samples.

Our observation of increasing SARS-CoV-2 viral load with increasing age, especially showing low viral loads in children $<12$ years of age, is not in line with all previous studies. ${ }^{5-8}$ Previous studies were all smaller and suffered from heterogeneity in the sampling technique, presence of symptoms, PCR technique (sometimes performed in different 
laboratories), symptom duration and test indication, and all included low numbers of paediatric patients. The largest of these-a cross-sectional study in Ghana including 9549 positive samples from both symptomatic and asymptomatic patients at various health facilities-showed the lowest median viral loads in those aged $\leq 10$ years $(n=280)$ and the highest in those aged $71-80$ years $(n=110)$, without further statistical analysis. ${ }^{9}$ The second largest study (4428 positive SARS-CoV-2 PCRs, including 96 patients born in or after 2000) did not show viral-load differences in various age brackets, although the presence and duration of symptoms were unknown and the study comprised all sorts of materials (NP, OP, BAL, nasal wash, etc.). ${ }^{5}$

The study of Jones et al., ${ }^{7}$ who analysed viral loads of 3712 patients (of all ages), reported no differences in viral load across age groups, although their study population included only 117 patients aged $<20$ years, and it was discussed by others that there was in fact moderate evidence of increasing viral load with increasing age present in their data. ${ }^{8}$ In our study, we had SARS-CoV-2 viral-load data available for 18290 unique patients tested in a Public Health setting, including 2654 patients aged $<20$ years (with 238 children aged $<12$ years). A study in 1213 positive specimens of symptomatic patients (with unknown symptom duration) only showed lower Ct-values in the $80-89$ years $(n=86)$ age group. ${ }^{10}$ A study in 1122 patients (type or setting unknown, tests performed in multiple laboratories) found a higher age in high-viral-load samples (Ctvalue <25: mean age 50 years) compared with moderate loads (Ct-value 25-30; mean age 48 years) and low viral loads (Ct-value $>30$, mean age 43 years) but, when stratifying per age group, the differences in high/moderate/low loads were not present. ${ }^{11}$ Other studies were much smaller, showing various results and as at least as much heterogeneity compared with these larger studies.

There are several potential explanations for the lower SARS-CoV-2 viral load in respiratory samples derived from children. For instance, the quality of the swabbing might differ between children and adults. The discomfort caused by nasopharyngeal or oropharyngeal sampling in children could have resulted in more nasal or midturbinate samples, despite clear testing guidelines. There are studies that showed combined throat/nasal-swab samples yielding a lower SARS-CoV-2 viral load compared with nasopharyngeal samples, ${ }^{12}$ although others showed no difference, ${ }^{13}$ or even higher viral loads in nasal or midturbinate samples. ${ }^{14}$ One could assume that even if the sampling method has a profound influence on the SARSCoV-2 viral load, this effect would have primarily been present in the youngest patients (where discomfort or protesting of the patient may have led to a different sampling procedure). As the effect of age on the SARS-CoV-2 viral load in our data is present across a broad range of age categories, we do not think this can be explained by differences in the sampling method alone. Moreover, to homogenize for the sampling method, we only included PCRs performed by Public Health Services (combined OP/NP performed by trained personnel). By only including Public Health PCRs, we also removed heterogeneity of inclusion criteria, although, for children, a restricted testing policy was employed during several months, where they needed to have severe symptoms (dyspnoea/fever) or a positive contact. Although it does explain the lower number of children tested, the increase in viral loads with age does not appear to change in time (Supplementary Table S4, available as Supplementary data at IJE online), so changes in the testing policy for children do not appear to explain our findings.

One could also argue that children were in general tested later after the first onset of symptoms compared with adults, as the parents postponed the moment of exposing their children to the distress of being sampled. This could have led to children being tested in a later stage of the infection, when their individual viral load may have declined compared with the early stage of the infections (following individual viral-load kinetics). ${ }^{2}$ However, results were similar when we adjusted the relationship between age and viral load for time since the onset of the disease.

Also, one could consider that, despite the need for symptoms to be included for testing by Public Health agencies before 1 December, some of the tested population might have falsely reported symptoms in order to be tested free of charge. It is impossible to correct for this potential bias, and it is impossible to ascertain whether there are age-dependent differences in this respect. Nevertheless, it seems unlikely that the observed large difference in Cp distribution is primarily caused by age-related differences in the false reporting of symptoms.

The observed lower viral load in children might be explained by age-related differences in viral-infection dynamics. For example, several studies have suggested a differential expression of angiotensin-converting enzyme 2 (ACE2) (the receptor that SARS-CoV-2 uses for host entry) in different age categories. ${ }^{15,16}$ Bunyavanich et al. showed a positive association between ACE2 gene expression and age, which might explain the lower incidence of COVID-19 in children and the lower SARS-CoV-2 viral loads that we found in the younger age categories. ${ }^{15}$ In addition, there are other factors that might protect children from higher viral load including e.g. differences in innate and adaptive immunity, more frequent recurrent and concurrent infections, pre-existing immunity to coronaviruses and differences in microbiota. ${ }^{17}$ 
Currently, antigen tests provide a rapid yet less sensitive method to diagnose SARS-CoV-2. Antigen tests do not employ a target replication technique and false-negatives are mostly observed in samples with a low viral load. ${ }^{18} \mathrm{We}$ found Cp-values of $>30$ in $31.1 \%$ of children aged $<12$ years, which was almost double the proportion found in the rest of the population. Therefore, these lower viral loads found in our study might indicate that antigen tests will have lower sensitivity in children. Although further studies should validate these findings, caution should be warranted when using antigen tests to diagnose SARS$\mathrm{CoV}-2$ in populations with lower viral loads, in order to reduce the risk of false-negative results.

One of the limitations of our study is that there were no data available on symptoms, underlying disease, the sampling method and the moment of onset of the first symptoms of all patients for whom respiratory samples were included. This is why the analyses of the relationship between age and viral load was evaluated in samples from patients tested in the Public Health testing facilities that was considered to be a relatively consistent population with respect to the performed sampling procedure and patient characteristics, and for whom the time of onset of the first symptoms was known (for a large subset of patients). Another limitation was the inclusion of symptomatic patients only, thus not reflecting the spectrum of SARSCoV-2 infection, especially as asymptomatic presentations are frequently seen in children. ${ }^{19-21}$

In addition, it should be noted that the samples included in this study were collected before the novel SARS-CoV-2 variant, VOC 202012/01, that was first identified in the UK was likely to have been widespread in the Netherlands. ${ }^{22}$

To conclude, with this study, we have tried to emphasize the usefulness of analysing SARS-CoV-2 RT-PCR (viralload) data that are derived from a large population made up of a broad range of patient groups and age categories in a single laboratory (using the same SARS-CoV-2 RT-PCR method for all samples). With these data, shifts in tested patient populations and viral-load distributions during the course of the COVID-19 pandemic can be closely monitored. This may contribute to a better understanding of SARS-CoV-2 transmission and improve future measures that are taken to restrict viral spreading. The most remarkable finding of this study was the relationship between SARS-CoV-2 viral load and age, with lower viral loads in children. As previous studies have suggested that young children $(<12$ years $)$ play a limited role in SARS-CoV-2 transmission, ${ }^{8,21,23}$ our data support this suggestion. Furthermore, these results suggest that SARS-CoV-2 antigen tests could have lower sensitivity in children than in adults.

However, viral load cannot solely explain the differences in the transmissibility between patients as e.g. epidemiological aspects (exposure to others) and clinical presentation (coughing as a symptom) should not be overlooked. ${ }^{6,8}$ In addition, other explanations such as the differences in the immune response in children and the effect of the test policy on the inclusion of children in our study population may have contributed to our findings. Further studies (combining viral-load data with contact-tracing data) should elucidate whether the lower viral load in children is indeed related to their suggested limited role in SARS-CoV-2 transmission.

\section{Supplementary data}

Supplementary data are available at IJE online.

\section{Ethics approval}

The Medical Ethical Committee of the Amsterdam UMC approved this study on 19 January 2021 (Study number: 2021.0022). In addition, the Institutional Review Board of the Spaarne Gasthuis, Hoofddorp/Haarlem, the Netherlands, approved the conduction of the study on 10 November 2020 (Study number: 2020.0154). The data were anonymized after collection and analysed under code.

\section{Funding}

No external funding was used for this study.

\section{Data availability}

The data underlying this article will be shared on reasonable request to the corresponding author.

\section{Acknowledgements}

We would like to thank all physicians, nurses, Public Health testing personnel, laboratory technicians and administration personnel who have worked hard to provide SARS-CoV-2 testing for a large number of individuals, making it possible to perform these analyses.

\section{Author contributions}

S.E., S.A., B.H., A.W. and D.S. participated in the conceptualization. S.E., I.M., B.H., J.S., J.K., R.J., I.L., C.G., D.S., J.C.S., F.S.M., J.B., A.W. and D.S. contributed to the data collection. S.E., A.W. and D.S. wrote the original draft. B.H., S.A., I.M., S.L., S.S.S., M.H., E.K., J.B. and E.S. contributed in reviewing and editing the paper. S.E., I.M. and D.S. performed the data curation and S.E. and D.S. contributed to the data analysis. All authors critically reviewed and approved the final version.

\section{Conflict of interest}

None declared. 


\section{References}

1. Corman VM, Landt O, Kaiser M et al. Detection of 2019 novel coronavirus (2019-nCoV) by real-time RT-PCR. Euro Surveill 2020;25:2000045.

2. Wölfel R, Corman VM, Guggemos W et al. Virological assessment of hospitalized patients with COVID-2019. Nature 2020; 581:465-73.

3. Jaafar R, Aherfi S, Wurtz N et al. Correlation between 3790 qPCR positives samples and positive cell cultures including 1941 SARS-CoV-2 isolates. Clin Infect Dis 2020;72:e921. https://doi. org/10.1093/cid/ciaa1491.

4. Rhoads D, Peaper DR, She RC et al. College of American Pathologists (CAP) Microbiology Committee perspective: caution must be used in interpreting the cycle threshold $(\mathrm{Ct})$ value. Clin Infect Dis 2020;72:e685-e686. 10.1093/cid/ciaa1199.

5. Kleiboeker S, Cowden S, Grantham J et al. SARS-CoV-2 viral load assessment in respiratory samples. J Clin Virol 2020;129: 104439.

6. Jacot D, Greub G, Jaton K, Opota O. Viral load of SARS-CoV-2 across patients and compared to other respiratory viruses. Microbes Infect 2020;22:617-21.

7. Jones TC, Mühlemann B, Veith T, et al. An analysis of SARSCoV-2 viral load by patient age. 2020. https://www.medrxiv. org/content/10.1101/2020.06.08.20125484v1 (1 June 2021, date last accessed).

8. Walsh KA, Jordan K, Clyne B et al. SARS-CoV-2 detection, viral load and infectivity over the course of an infection. J Infect 2020; 81:357-71.

9. Owusu M, Sylverken AA, Ankrah ST et al. Epidemiological profile of SARS-CoV-2 among selected regions in Ghana: a cross-sectional retrospective study. PLoS One 2020;15: e0243711. https://doi.org/10.1371/journal.pone.0243711.

10. Buchan B, Hoff JS, Gmehlin CG et al. Distribution of SARSCoV-2 PCR cycle threshold values provide practical insight into overall and target-specific sensitivity among symptomatic patients. Am J Clin Pathol 2020;154:479-85.

11. Maltezou HC, Raftopoulos V, Vorou R et al. Association between upper respiratory tract viral load, comorbidities, disease severity and outcome of patients with SARSCoV-2 infection. J Infect Dis 2021;223:1132-1138. https://academic.oup.com/ jid/advance-article/doi/10.1093/infdis/jiaa804/6060070.
12. Vlek ALM, Wesselius TS, Achterberg R, Thijsen SFT. Combined throat/nasal swab sampling for SARS-CoV-2 is equivalent to nasopharyngeal sampling. Eur J Clin Microbiol Infect Dis 2021; 40:193-95.

13. Péré $H$, Podglajen I, Wack $M$ et al. Nasal swab sampling for SARS-CoV-2: a convenient alternative in times of nasopharyngeal swab shortage. J Clin Microbiol 2020;58:e0721-20.

14. Tu YP, Jennings R, Hart B et al. Swabs collected by patients or health care workers for SARS-CoV-2 testing. NEJM 2020; 383:5.

15. Bunyavanich S, Do A, Vicencio A. Nasal gene expression of angiotensin-converting enzyme 2 in children and adults. JAMA 2020;323:2427-29.

16. Patel AB, Verma A. Nasal ACE2 levels and Covid-19 in children. JAMA 2020;323:2386-87.

17. Zimmermann P, Curtis N. Why is COVID-19 less severe in children? A review of the proposed mechanisms underlying the age-related difference in severity of SARS-CoV-2 infections. Arch Dis Child 2021;106:429-39.

18. Porte L, Legarraga P, Vollrath V et al. Evaluation of a novel antigen-based rapid detection test for the diagnosis of SARS-CoV-2 in respiratory samples. Int J Infect Dis 2020;99: 328-33.

19. Baggio S, L'Huillier AG, Yerly $S$ et al. SARS-CoV-2 viral load in the upper respiratory tract of children and adults with early acute COVID-19. Clin Infect Dis 2020;73:148-150; doi: 10.1093/cid/ciaa1157.

20. Zimmermann P, Curtis N. COVID-19 in children, pregnancy and neonates: a review of epidemiologic and clinical features. Pediatr Infect Dis J 2020;39:469-77.

21. Williams PCM, Howard-Jones AR, Hsu P et al. SARS-CoV-2 in children: spectrum of disease, transmission and immunopathological underpinnings. Pathology 2020;52:801-08.

22. Davies NG, Barnard RC, Jarvis CI, et al. Estimated Transmissibility and Severity of Novel SARS-CoV-2 Variant of Concern 202012/01 in England. 2020. https://www.medrxiv. org/content/10.1101/2020.12.24.20248822v1 (1 June 2021, date last accessed).

23. Gudbjartsson DF, Helgason A, Jonsson $\mathrm{H}$ et al. Spread of SARSCoV-2 in the Icelandic population. N Engl J Med 2020;382: 2302-15. 